\title{
Percutaneous Endoscopic Gastrostomy in Children: Experience from Single Center of a Developing Country
}

\author{
Kehar $\mathbf{M}^{1}$, Upadhyay $\mathbf{S}^{2}$, Wadhwa $\mathbf{N}^{3}$
}

\begin{abstract}
Introduction: Nutrition is of paramount importance for adequate growth and development of a child. Various routes of providing enteral nutrition to a paediatric patient are by nasogastric, nasojejeunal and gastrostomy which can be placed surgically or endoscopically. The objectives of this study were to review cases with percutaneous endoscopic gastrostomy (PEG) procedure and patient characteristics, indications, complications and outcome of PEG tube insertion in children at our center. Materials and Methods: This was a prospective study carried out in Sir Ganga Ram Hospital in New Delhi, India for a period of two years from August 2010 to August 2012. It included patients in whom PEG tube were placed during the study period and have had at least one year of post procedure follow up. Demograhic details, duration of procedure, complications, initial weight and height and then at 3 month, 6 months and 12 months of PEG tube placement were also recorded. Data between groups was compared using ANOVA and within groups across follow-ups was done using paired t-test. Results: Fourty six PEG insertions were performed during the study period, 26 twenty six conversions to BRT or Mickey button and ten PEG removals. The main indications for PEG insertion were Cerebral palsy with feeding difficulty $(47.8 \%)$. Erythema at the PEG insertion site was the most common complication (21\%). There was significant improvement in the weight and height in all age group of patients at 3, 6 and 12 months post procedure with a $p$ value $<0.5$. The average weight gain after 3,6 and 12 months was $1.3 \mathrm{~kg}, 2.8 \mathrm{~kg}$ and $4.2 \mathrm{~kg}$ and the average height gain after 3,6 and 12 months was $1.6 \mathrm{~cm}, 2.5 \mathrm{~cm}$ and $4.13 \mathrm{~cm}$ respectively. Conclusions: PEG is effective means for optimizing the nutritional goals of patients who are nutritionally debilitated with minimal complications.
\end{abstract}

Key words: Percutaneous endoscopic gastrostomy, Enteral nutrition.

\section{Introduction}

$\mathrm{N}$ utrition is of paramount importance for adequate growth and development of a child. Enteral nutrition is the ideal mode of nutrient delivery for children ${ }^{1,2}$ The various routes of providing enteral nutrition to a paediatric patient are by nasogastric tube
'Dr. Mohit Kehar, MBBS, DNB Paediatrics, Fellowship in Paediatric Gastroenterology and Hepatology, Registrar, '2Dr. Satyam Upadhyay, MBBS, MD, Fellow division of Paediatric Gastroenterology and Hepatology, ${ }^{3}$ Dr. Nishant Wadhwa, MBBS, DCH, DNB, Consultant and Chief. All from the Division of Paediatric Gastroenterology and Hepatology, Institute of Child Health, Sir Ganga Ram Hospital, Delhi.

\section{Address for correspondence:}

Dr. Mohit Kehar

Registrar, Division of Paediatric Gastroenterology and Hepatology,

Institute of Child Health,

Sir Ganga Ram Hospital, Delhi.

E-mail: mohitkehar86@yahoo.co.in

Tel No: +91 9810266452

\section{How to cite}

Kehar M, Upadhyay S, Wadhwa N. Percutaneous Endoscopic Gastrostomy in Children: Experience from Single Center of a Developing Country. J Nepal Paediatr Soc 2014;34(2):125-131.

doi: http://dx.doi.org/10.3126/jnps.v34i2.10960

This work is licensed under a Creative Commons Attribution 3.0 License.

\section{(c) (i)}

insertion, nasojejeunal tube insertion, gastrostomy which can be placed surgically or endoscopically.

Nasogastric tube feeding is most often used for short-term periods, however, there are several limitations for its long-term use including nasal discomfort, blockage or displacement of the tube, irritation or penetration of the larynx and recurrent pulmonary aspirations ${ }^{3}$. 
Gastrostomy has various advantages over nasogastric tube as there is less chance of blocking and tube displacement and these tubes donot require frequent revisions. Gastrostomy tubes can be placed surgically or endoscopically. The superiority of percutaneously placed gastrostomies compared to former surgical gastrostomy procedures (i.e. Witzel, Stamm, Janeway techniqueues) has been shown clearly in many clinical studies ${ }^{4,5}$. For these obvious reasons, percutaneous endoscopic gastrotomy (PEG) is now the preferred option for providing medium and longterm nutritional support in patients with impairment of feeding abilities leading to under nutrition and its complications.

PEG was first introduced by Gauderer in 1980, the first PEG insertion was performed in University hospital of Cleveland, USA, on a four and-half monthold baby ${ }^{6}$. In addition to improving the nutrition and growth of subjects, the use of the PEG can significantly reduce feeding time and ease drug administration. There has been a consistent improvement in the social functioning, mental, general health perception, and quality of life of caregivers in prospective cohort studies $^{7}$. It has been found in various studies that the nutritional status of unwell children is a common cause of anxiety for parents and feeding times can be stressful ${ }^{8}$.

The impact of PEG feeding is positive with many parents reporting a high level of satisfaction and wishing the intervention to have taken place earlier ${ }^{9,10}$. Nutritional support with the use of the PEG has been demonstrated in children with neurodisability ${ }^{10,11}$, cystic fibrosis ${ }^{12,13}$, neonatal pulmonary disease ${ }^{14}$, congenital heart disease $(\mathrm{CHD})^{15,16}$, Crohn disease ${ }^{17}$, oncological conditions ${ }^{18}$, metabolic disease, genetic chromosomal, and degenerative diseases ${ }^{19}$. PEG is widely being used for paediatric patients in developed countries for various indications.

The aim of our study were to review cases with percutaneous endoscopic gastrostomy (PEG) procedure and to review patient characteristics, indications, and complications and outcome of PEG tube insertion in children at our center.

\section{Materials and Methods}

The study was a prospective study carried out in one of the tertiary care hospitals, Sir Ganga Ram Hospital, Division of Paediatric Gastroenterology and Hepatology, New Delhi, India for a period of two years from August 2010 to August 2012.
All patients who underwent PEG tube placement and have had at least one year of follow up were enrolled for the study after informed consent from parents/guardians of the patients. As exclusion criteria all patients who had less than one year of follow up after PEG placement were excluded from the study. Patients with acute systemic illness, with deranged coagulation profile or/ and thrombocytopenia, were also excluded for PEG placement.

The study was approved from ethical committee as per hospital research committee protocol. Patient demographics, principal diagnosis and indication for PEG placement, length of hospital stay after PEG and post procedure complications were recorded for all the patients from the medical charts. The patients were followed up and weight gain and height gain was periodically checked after 3 months, 6 months and 12 months of PEG placement.

PEG placements were performed under moderate sedation using Midazolam and Ketamine in the endoscopy suite by a team consisting of a pediatric gastroenterologist and accompanying team. Standard "pull technique" was used for all PEG placements. As per protocol all patients underwent a pre procedure work up consisting of a complete hemogram and coagulation profile.

All patients received a preoperative single dose of antibiotic a third generation cephalosporin, immediately before PEG placement. Kimberley clark / Freka PEG tubes were used of size 14- $24 \mathrm{~F}$ depending on the age and weight of the patient. In patients aged 0-1 yr 14 Fn tube was used, in 1-6 yrs 20Fn tube and in older than 6 yrs $24 \mathrm{Fn}$ tube was used to provide enteral nutrition support. After the PEG tube placement, feeds were initiated after 4-6 hrs of procedure and parents were explained the feeding process and handling of the tube.

All patients were started on measure to reduce gastroesophageal reflux like head end elevation and proton pump inhibitors for at least three months. After this they were continued on positional measures and PPI were stopped and the patients were followed up for symptoms and signs of reflux.

Permanent PEG removals were performed when children no longer required the PEG for feeding support as decided following a detailed multidisciplinary assessment which includes a detailed dietary history assessing the optimum calories, macronutrients and drugs the patient is having orally along with 
extend of the recovery of primary illness for which PEG tube was placed. PEG removal was performed endoscopically under sedation. Whenever long term support was required PEG tube was replaced with a Balloon replacement tube (BRT) or Mickey button after 3-5 months of primary tube insertion. Tube was replaced because of wear and tear in the tube; it was either blocked or disfigured so it was replaced with a replacement tube.

The patients were divided in four groups according to the age of patient ie 0-1 yr, 1.1-5 yr, 5.1-10 year and 10.1-16 years and the follow up weight and height was compared in all the four groups. Data analysis between groups has been compared using ANOVA and within groups across follow-ups has been done using paired t-test. SPSS 15.0 .

\section{Results}

Forty six PEG tube placements were performed during the study period. Amongst the patient, 30 were male and 16 were females. The median age of patients at time of PEG insertion was 6.75 years (range is 5 weeks - 16 years) and mean weight was $10.3 \mathrm{~kg}$ (range $2.4 \mathrm{~kg}$ - $40 \mathrm{~kg}$ ). The commonest indication for PEG insertion were Cerebral palsy (CP) (47.8\%) and failure to thrive with feeding difficulty and recurrent aspirations (13\%) followed by congenital heart disease requiring adequate weight gain (8.6\%). (Table 1 )

Table 1: Indication for PEG tube placement

\begin{tabular}{|l|c|c|}
\hline Indication & No & $\%$ \\
\hline Cerebral palsy & 22 & 47.8 \\
\hline Feeding difficulty & 6 & 13 \\
\hline Congenital heart disease & 4 & 8.6 \\
\hline Head injury & 3 & 6.5 \\
\hline Tubercular meningitis & 2 & 4.3 \\
\hline Intraventricular bleeding & 2 & 4.3 \\
\hline Polytrauma & 2 & 4.3 \\
\hline Medulloblastoma & 2 & 4.3 \\
\hline Rett syndrome & 1 & 2.1 \\
\hline Gullein Barre syndrome & 1 & 2.1 \\
\hline Duchenne muscular dystrophy & 1 & 2.1 \\
\hline
\end{tabular}

Three patients had head injury with diffuse neuronal involvement (6.5\%), two patients were suffering from tubercular meningitis with hydrocephalus (4.3\%). There were two patients each suffering from polytrauma and intraventricular bleeding (4.3\%). There were two patients of medulloblastoma (4.3\%), and one patient each of Retts syndrome, Duchenne muscular dystrophy, and Gullein Barre syndrome. (2.1\% each)
The youngest patient was a five week old male child who was suffering from retrognathia with cleft palate with feeding difficulty with recurrent aspirations whose weight at the time of PEG tube placement was $2.4 \mathrm{~kg}$. Our series includes ten infants, the oldest being nine month old at the time of PEG insertion.

The time for PEG placement was SD 14.39+_4.14 range 10-20 minute). The duration of hospital stay following PEG insertion was one day with a range of one to four days. No patient remained in hospital beyond this time having had a PEG as the sole procedure

Erythema at the PEG insertion site was the most common complication post procedure (10/46, $21 \%)$. In all instances this was treated with oral antibiotics for 5 days with no progression. Two patients had reflux of feeds from the PEG tube which improved after giving laxatives as both these patient were suffering from chronic constipation. No procedure-related mortality was noticed.

Thirty six children underwent PEG removal during the study period. These are patients in whom either PEG tube was changed with a BRT or in whom it was not required anymore so it was removed permanently. In twenty six patients, the PEG was substituted with $\mathrm{BRT} /$ mickey button, whereas in ten children the PEG was removed because it was no longer required for feeding support. The average duration between the PEG insertions to BRT conversion was 4.7 months.

Over the study period ten patients had the PEG permanently removed because it was no longer required for feeding support (average duration after PEG insertion 1.3 years). Out of these ten patients, two were suffering from tubercular meningitis, two with head injury, two with intracranial bleeding, two patient of polytrauma, one patient each of medulloblastoma and Gullein Barre syndrome. No patient had PEG removal before one year of tube placement.

All PEG tube removals were done endoscopically under sedation with no complications. Traction technique for tube removal was not used in any of the patient as the tubes used in our unit were not traction removable. In follow up out of forty six patients in whom PEG tube was placed, ten patients lost to our follow up and rest thirty six children were under follow up for atleast one year during which there weight and height were measured. The ten patients who were lost to follow up included four patients with cerebral palsy, two patients with feeding difficulty with failure to thrive, one patient suffering from head injury, one patient with patent ductus arteriosus with severe 
failure to thrive, one patient of medulloblastoma and one patient of Rett syndrome.

Three patients had an increase in amount of reflux following PEG tube placement, which manifested as recurrent apneic attack in one patient who was admitted in neonatal intensive care unit suffering from hypoxic ischemic encepathalopathy sequel with cleft lip and palate. The other two patients had repeated vomiting and aspiration pneumonia. For these patients a feeding tube was guided endoscopically through the PEG tube feeding channel into the jejunum under fluoroscopy (Jejunal tube percutaneous endoscopic gastrotomy /JET -PEG). Post placement of JET-PEG, there was consistent weight gain and improvement in reflux symptoms in these patients.

There were thirty six patients who were under follow up for one year. After three month of PEG tube placement average weight gain noticed was $1.3 \mathrm{~kg}$ and height gain of $1.6 \mathrm{~cm}$. After six months the average weight gain was $2.8 \mathrm{~kg}$ with height gain of $2.5 \mathrm{~cm}$, after twelve average weight gain was $4.2 \mathrm{~kg}$ and height gain of $4.13 \mathrm{~cm}$ (Fig 1).

As the growth velocity is different in different age group of patients, four age group were formed and the average weight and height of all these 4 groups were compared.

In all the four groups there was consistent height and weight gain at 3,6 and 12 months with $p<0.05$ in all groups when there average weight and height was compared with their presentation parameters (Table 2 and 3).

In age group 0-1 yr the percentage improvement in height and weight at 3, 6 and 12 months was best amongst the entire four groups owing to high growth velocity in this age group of patients. (Figure 1, 2).

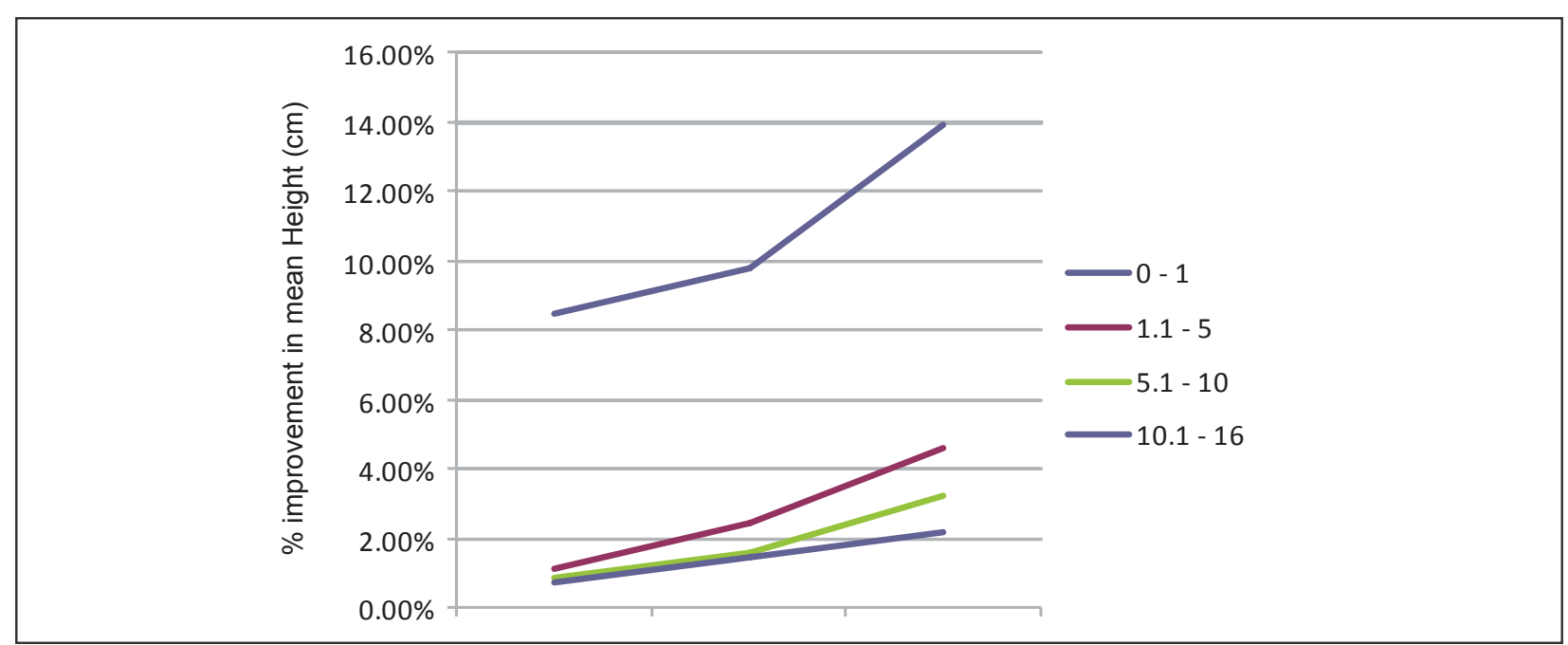

Fig 1: Percentage weight improvement in different age groups at 3, 6 and 12 months after PEG insertion

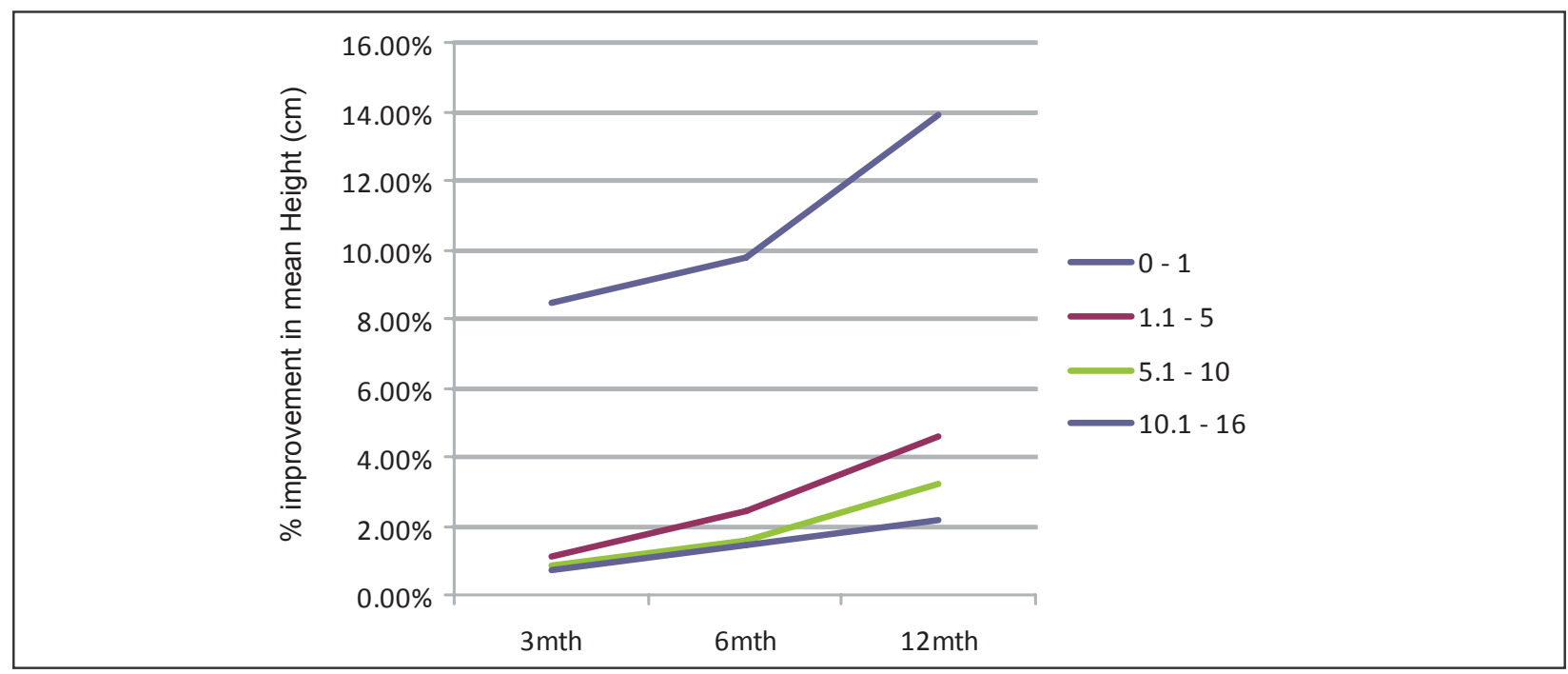

Fig 2: Percentage height improvement in different age groups at 3, 6 and 12 months after PEG insertion 
Table 2: Mean weight 3, 6 and 12 months with $p$-values of different age groups

\begin{tabular}{|c|c|c|c|c|c|c|c|c|}
\hline \multirow{2}{*}{$\begin{array}{c}\text { Age } \\
\text { Interval }\end{array}$} & \multirow{2}{*}{$\mathbf{N}=36$} & \multicolumn{4}{|c|}{ Weight (kg) } & \multicolumn{3}{c|}{$p$-values (presentation Vs.) } \\
\cline { 3 - 9 } & & Presentation & $\mathbf{3}$ months & $\mathbf{6}$ months & $\mathbf{1 2}$ months & 3 months & $\mathbf{6}$ months & $\mathbf{1 2}$ months \\
\hline $0-1$ & 7 & $3.81 \pm 1.34$ & $6.74 \pm 1.4$ & $8.71 \pm 1.15$ & $10.49 \pm 1.37$ & $<0.001$ & $<0.001$ & $<0.001$ \\
\hline $1.1-5$ & 8 & $12.2 \pm 2.43$ & $13.23 \pm 2.45$ & $15.09 \pm 2.29$ & $16.01 \pm 2.17$ & $<0.001$ & $<0.001$ & $<0.001$ \\
\hline $5.1-10$ & 9 & $20.51 \pm 5.73$ & $21.57 \pm 5.92$ & $22.79 \pm 5.49$ & $23.99 \pm 5.52$ & $<0.001$ & $<0.001$ & $<0.001$ \\
\hline $10.1-16$ & 12 & $30.97 \pm 5.91$ & $31.76 \pm 5.89$ & $32.95 \pm 5.98$ & $34.56 \pm 5.94$ & $<0.001$ & $<0.001$ & $<0.001$ \\
\hline Total & $\mathbf{3 6}$ & $\mathbf{1 8 . 9} \pm \mathbf{1 1 . 2 4}$ & $\mathbf{2 0 . 2 3} \pm \mathbf{1 0 . 6 8}$ & $\mathbf{2 1 . 7 3 \pm 1 0 . 3 4}$ & $\mathbf{2 3 . 1 1 \pm 1 0 . 3 8}$ & & & \\
\hline
\end{tabular}

Table 3: Mean height 3, 6 and 12 months with $P$ values of different age groups

\begin{tabular}{|c|c|c|c|c|c|c|c|c|}
\hline \multirow{2}{*}{$\begin{array}{c}\text { Age } \\
\text { Interval }\end{array}$} & \multirow{N}{*}{$\mathbf{N} \mathbf{3 6}$} & \multicolumn{4}{|c|}{ Height (cms) } & \multicolumn{2}{c|}{$p$-values (presentation Vs.) } \\
\cline { 3 - 9 } & & Presentation & $\mathbf{3}$ months & $\mathbf{6}$ months & $\mathbf{1 2}$ months & $\mathbf{3}$ months & $\mathbf{6}$ months & $\mathbf{1 2}$ months \\
\hline $0-1$ & 7 & $55.57 \pm 6.9$ & $60.29 \pm 6.68$ & $61 \pm 6.89$ & $63.29 \pm 6.05$ & 0.001 & $<0.001$ & $<0.001$ \\
\hline $1.1-5$ & 8 & $86 \pm 5.81$ & $86.94 \pm 5.5$ & $88.06 \pm 5.54$ & $89.94 \pm 5.78$ & $<0.001$ & $<0.001$ & $<0.001$ \\
\hline $5.1-10$ & 9 & $110 \pm 12.61$ & $110.94 \pm 12.46$ & $111.72 \pm 12.74$ & $113.5 \pm 12.55$ & $<0.001$ & $<0.001$ & $<0.001$ \\
\hline $10.1-16$ & 12 & $128.58 \pm 7.77$ & $129.5 \pm 7.73$ & $130.42 \pm 7.67$ & $131.38 \pm 8.1$ & $<0.001$ & $<0.001$ & $<0.001$ \\
\hline Total & $\mathbf{3 6}$ & $\mathbf{1 0 0 . 2 8} \pm \mathbf{2 8 . 5 6}$ & $\mathbf{1 0 1 . 9 4} \mathbf{2 7 . 3 4}$ & $\mathbf{1 0 2 . 8 3} \pm \mathbf{2 7 . 4}$ & $\mathbf{1 0 4 . 4 6 \pm \mathbf { 2 6 . 9 5 }}$ & & & \\
\hline
\end{tabular}

\section{Discussion}

Gastrostomy tubes were placed exclusively by laparotomy until 1980, when Gauderer showed that the PEG technique was more cost-effective and safer than surgical gastrostomy ${ }^{6}$. PEG technique is better because it avoids the morbidity associated with laparotomy, causes less incisional pain, has a quicker recovery period, and can be performed more rapidly, with the average PEG taking less than 15 minutes $^{20}$.

Neurodisabilty was the main indication for PEG insertion in our experience. Cerebral palsy was the single most important indication for PEG insertion. Craig et al have reported PEG experience in a North London cohort where the predominant indication for insertion of PEG was CP followed by genetic syndromes, metabolic syndromes, and progressive degenerative disorder ${ }^{21}$. Feeding difficulty was the main indication for PEG insertion in a South African series ${ }^{22}$, whereas neuromuscular and metabolic cause ${ }^{23}$ and faltering growth ${ }^{24}$ were the most important indication in other studies.

There was consistent weight and height gain in all age group patients after PEG insertion with best percentage was seen in patients aged less than one year owing to their normal high growth velocity.

In our study, no major complication was seen and there were no procedure related mortality. Only minor complication were seen which was erythema of the skin, similar complications were also reported by a group from Liverpool, United Kingdom ${ }^{25}$.
In our series following 46 new PEG tube placements three patient $(6.5 \%)$ had increase in amount of reflux manifesting as apnea, vomiting and aspiration pneumonia. In all these cases a JET PEG was done following which there was improvement in symptoms and growth velocity. There have been conflicting studies on the risk of gastroesophageal reflux after PEG tube placement however, the role of PEG as a cause of new onset gastoesophageal reflux in children remains controversia|26,27. We have not studied this parameter systemically, but as our unit protocol, all patients following PEG tube placement are placed on measures to prevent reflux.

The duration for PEG tube feeding depends upon the indication for which it was placed; patients suffering from cerebral palsy require long term support for optimal nutrition. Patients suffering from GBS, polytrauma, TBM require short term support owing to recovery of their neurological insult.

\section{Conclusion}

There is an increasing demand for PEGs in management of chronic pediatric ailments who have nutritional challenge and when feeding problems become a hindrance in growth and development. There has been a consistent weight gain in the patients on PEG tube, as calorie dense feeds can be given easily which would otherwise not be possible with a nasogastric tubes for prolonged periods. Our complication rates compare favorably with those reported in other series with minor complications like local skin infections easily amenable to treatment. 
Acknowledgements: Nil

Funding: Nil

Conflict of Interest: Nil

Permission from IRB: Yes

\section{References}

1. Wildhaber BE, Yang $H$, Spencer $A U$, Drongowski RA, Teitelbaum DH.. Lack of enteral nutrition effects on the intestinal immune system. J Surg Res 2005;123:8-16.

2. Kudsk K. Effect of route and type of nutrition on intestine-derived inflammatory response. Am J Surg 2003;185:16-21.

3. Holmes S. Enteral feeding and percutaneous endoscopic gastrostomy. Nurs Stand 2004;18:41-3.

4. Ho CS, Yee ACN, McPherson R. Complications of surgical and percutaneous nonendoscopic gastrostomy: review of 233 patients. Gastroenterology 1988;95(5):1206-10

5. Grant JP. Comparison of percutaneous endoscopic gastrostomy with Stamm gastrostomy. Annal Surg 1988;207:598-603.

6. Gauderer MWL, Ponsky JL, Izant RJ. Gastrostomy without laparoscopy-a percutaneous endoscopic technique. J Pediatr Surg 1980;15:872

7. Sullivan $P B$, Juszczak $E$, Bachlet $A M$, Thomas AG, Lambert B, Vernon-Roberts A, etal. Impact of gastrostomy tube feeding on the quality of life of carers of children with cerebral palsy. Dev Med Child Neurol 2004;46:796-800

8. Sloper P, Turner S. Risk and resistance factors in the adaptation of parents of children with severe physical disability. J Child Psychol Psychiatr 1993;34:167-88.

9. Avitsland $\mathrm{TL}$, Kristensen C, Emblem R, Veenstra M, Mala T, Bjørnland K. Percutaneous endoscopic gastrostomy in children: a safe technique with major symptom relief and high parental satisfaction. J Pediatr Gastroenterol Nutr 2006;43:624-8.

10. McGrath SJ, Splaingard ML, Alba HM, Kaufman $\mathrm{BH}$, Glicklick M. Survival and functional outcome of children with severe cerebral palsy following gastrostomy. Arch Phys Med Rehabil 1992;73:1337.

11. Sullivan PB, Juszczak E, Bachlet AM, Lambert B, Vernon-Roberts A, Grant HW, etal. Gastrostomy tube feeding in children with cerebral palsy: a prospective, longitudinal study. Dev Med Child Neurol 2005;47:77-85.
12. Richter T, Meier C, Steppberger K, Knorrek G, Lietz T. Experiences with enteral nutrition of patients with cystic fibrosis (CF) via a percutaneous endoscopic gastrostomy (PEG). Klin Padiatr 2001;213:325-8.

13. Williams SG, Ashworth F, McAlweenie A, Poole $S$, Hodson ME, Westaby D. Percutaneous endoscopic gastrostomy feeding in patients with cystic fibrosis. Gut 1999;44:87-90.

14. Guimber D, Michaud L, Storme L, Deschildre A, Turck D, Gottrand F. Gastrostomy in infants with neonatal pulmonary disease. I Pediatr Gastroenterol Nutr 2003;36:459-63.

15. Ciotti G, Holzer R, Pozzi M, Dalzell M. Nutritional support via percutaneous endoscopic gastrostomy in children with cardiac disease experiencing difficulties with feeding. Cardiol Young 2002;12:537-41.

16. Hofner G, Behrens R, Koch A, Singer H, Hofbeck M.. Enteral nutritional support by percutaneous endoscopic gastrostomy in children with congenital heart disease. Pediatr Cardiol 2000;21:341-6.

17. Cosgrove M, Jenkins HR. Experience of percutaneous endoscopic gastrostomy in children with Crohn's disease. Arch Dis Child 1997;76:1413.

18. Skolin I, Hernell O, Larsson MV, Wahlgren C, Wahlin YB. Percutaneous endoscopic gastrostomy in children with malignant disease. J Pediatr Oncol Nurs 2002;19:154-63.

19. Casswall T, Backstrom B, Drapinski M, et al. Help to children and adolescents with malnutrition or eating disorders. Percutaneous endoscopic gastrostomy with button: simple safe and costeffective. Lakartidningen 2000;97:688-91.

20. Ljungdahl $M$, Sundbom M. Complication rate lower after percutaneous endoscopic gastrostomy than after surgical gastrostomy: a prospective, randomized trial. Surg Endosc 2006;20:1248-51

21. Craig GM, Carr LJ, Cass $H$, Hastings RP, Lawson $M$, Reilly $S$, etal. Medical, surgical, and health outcomes of gastrostomy feeding. Dev Med Child Neurol 2006;48:353-60

22. Van der Merwe WG, Brown RA, Ireland JD, Goddard E. Percutaneous endoscopic gastrostomy in children-a 5-year experience. S Afr Med J 2003;93:781-5.

23. Yeh TC, Yeung CY, Sheu JC, Lee HC, Lin SP, Hsu $\mathrm{CH}$, Lee $\mathrm{YJ}$, etal. Percutaneous endoscopic gastrostomy in children: 15 cases experience. Acta Paediatr Taiwan 2003;44:135-39. 
24. Eger R, Reif S, Yaron A, Bojanover Y. Percutaneous endoscopic gastrostomy (PEG) in children: indications, the procedure, outcomes, short and long-term complications. Harefuah 2008;147:214.

25. Srinivasan $R$, Irvine $T$, Dalzell $M$. Indications for Percutaneous Endoscopic Gastrostomy and Procedure-related outcome. J Pediatr Gastroenterol Nutr 2009;49:584-88.
26. Behrens $R$, Lang $T$, Muschweck $H$, Richter T, Hofbeck M. Percutaneous endo- scopic gastrostomy in children and adolescents. J Pediatr Gastroenterolgy Nutr 1997;25:487-91.

27. Fox VL, Abel SD, Malas S, Duggan C, Leichtner AM. Complications following percutaneous endoscopic gastrostomy and subsequent catheter replacement in children and young adults. Gastrointest Endosc 1997;45:64-71. 